\title{
EL ESPIRITU DE LA RISA EN EL CUENTO DE ANA LYDIA VEGA
}

POR

\author{
YVONNE CAPTAIN-HIDALGO \\ George Washington University
}

El humor es una de las características estructurantes de la cuentística de Ana Lydia Vega. Aunque en la crítica literaria en general se ha investigado mucho este aspecto de la literatura, ninguno de los estudios de la obra de Vega se basa en ello. Es como si los críticos creyeran el viejo patrón, establecido desde Aristóteles y continuado hasta nuestros días: lo cómico existe solamente con el fin de hacernos reír y de pintar un cuadro de los seres mal ajustados a la sociedad y no dignos de un retrato trágico:

Comedy [...] is an imitation of persons worse than the average. Their badness, however, does not extend to the point of utter depravity; rather, ridiculousness is a particular form of the shameful and may be described as the kind of error and unseemliness that is not painful or destructive (Aristotle, 49).

Mi propósito es llegar a la profundidad del humor en la obra de Vega y sacar algunas conclusiones sobre adónde lleva al lector. Por lo general los cuentos son variados en su tratamiento de la condición del ser humano. Van de lo más íntimo a lo muy colectivo. Se expresan tan bien en forma de alegoría como de narración directa. Lo que más tienen en común en cuanto a su expresividad es su variado sentido de humor.

Comedia, parodia, sátira e ironía, lo ridículo, faceto, absurdo y risible todos caracterizan la cuentística de Vega. Nada más falta una tipología para determinar su nivel de risa. En este momento, sin embargo, me interesan más esos cuentos de risa y los recursos poéticos empleados por Vega para lograr ese efecto.

En su proyección más amplia, la risa, según la antropología, es una de las características de todos los seres humanos (Apte). En este sentido cabe muy bien un análisis bakhtiano, muy de moda hoy en día, en que la subversión temporal del orden social ayuda a purificar lo serio:

True, ambivalent and universal laughter does not deny seriousness, but purifies and completes it. Laughter purifies from dogmatism, from the intol- 
erant and the petrified; it liberates from fanaticism and pedantry, from fear and intimidation, from didacticism, naïvete and illusion from the single meaning, the single level, from sentimentality. Laughter does not permit seriousness to atrophy and to be torn away from the one being, forever incomplete. It restores this ambivalent wholeness. Such is the function of laughter in the historical development of culture and literature (Bakhtin, 122-123).

Del ejemplo bakhtiano sacamos una base teórica para apoyar las ideas sobre el humor en la obra de Vega. También de Bakhtin logramos un estudio del poder -los que lo tienen y los que quedan sin él. Seguramente el cuento de la puertorriqueña se trata de diferencias entre clases sociales igual que en la sociedad medieval de los terratenientes y los peones, según analiza Bakhtin en su estudio sobre lo carnavalesco.

Sin embargo, debe haber otras proyecciones aún más específicas de lo universal que leemos en Bakhtin - proyecciones que enmarcan los cuentos de Vega, según el contexto sociopolítico de que salen. Una proyección muy poco mencionada, pero que se ve y se vive en la obra, es la dimensión transnacional de la opresión. En su punto de vista de los de abajo, los narradores y personajes revelan un mundo jamás imaginado por la muy-amplia teoría literario-cultural de Bakhtin. Algunos han empezado a analizar el aspecto particular y serio del humor en Latinoamérica.

Latin American literature [...] belong[s] to a venerable Latin American tradition of laughter. Present in our literature from its origins, laughter is the weapon the oppressed use to parody and destroy the solemnity of their oppressors [...] For these authors, parody and satire are weapons of self-defense and attack they use against a society whose laws were perfect in theory and outrageous in practice (Rodriguez Monegal, 3). ${ }^{1}$

De igual modo que el análisis baktiano no explica el total del humor en Vega, tampoco sirve totalmente esta más especifica crítica sobre el humor en la literatura hispanoamericana, caribeña o puertorriqueña que ofrece Rodríguez Monegal. Sin duda es, como explica Rodríguez Monegal - la realidad según una historia, y unas circunstancias bien específicas. Pero al mismo tiempo, a veces es necesario salir de un mundo lingüístico- $\mathrm{y}-$ culturalmente particular. $\mathrm{Al}$ eliminar las palabras "Latin American" y al sustituir ejemplos de otros sectores del mundo, el lector discierne una nueva base con que trabajar el humor en la obra de Vega.

El mundo al que me refiero es habitado por los que ni son practicantes ni satélites de los superpoderes y se caracteriza por lo que no tienen. El término "tercer mundo" sigue siendo aplicable para agrupar a las naciones y subculturas que carecen de recursos económicos (Wolf-Phillips). Aún teniendo en cuenta la estratificación económico-social entre los mismos tercermundistas, hay ligaciones

${ }^{1}$ I wish to thank the poet and critic Roberto Valero for sharing this article with me. 
innegables. En el caso de la clase alta de esas mismas sociedades, todavía se puede considerar tercermundistas a los que tienen los ojos siempre hacia Europa o los Estados Unidos como su punto de referencia cultural. Si la crítica literaria en Hispanoamérica de la década de los ochenta se caracteriza principalmente por un rechazo de conceptos de diferenciación basados en lo económico y hasta en lo racial - a favor de una visión global, curiosamente, el "globo" se limitaba a una proyección eurocéntrica, olvidando que lo realmente global en sí puede comprender varias manifestaciones de cultura. Una de éstas a que el crítico literario necesita acudir es la literatura de las naciones pobres - literatura que muchas veces focaliza conscientemente la globalidad de los oprimidos raciales. Es una división económica, como señala Bakhtin, y es cultural, como nos recuerda Rodríguez Monegal. Pero a la vez difiere, porque ya no se trata de los pobres dentro de un pueblo, sino de naciones pobres frente a las ricas y poderosas. Además, como explica Harlow, es una división históricopolítica, basada en una resistencia consciente:

The struggle for national liberation and independence, particularly in the twentieth century, on the part of colonized peoples in those areas of the world over which Western Europe and North America have sought socio-economic control and cultural dominion has produced a significant corpus of literary writing, both narrative and poetic [sic], as well as a broad spectrum of theoretical analyses of the political, ideological, and cultural parameters of this struggle. This literature, like the resistance and national liberation movements which it reflects and in which it can be said to participate, not only demands recognition of its independent status and existence as literary production, but as such also presents a serious challenge to the codes and canons of both the theory and the practice of literature and its criticism as these have been developed in the West (Harlow).

Hasta cierto punto se trata de lo que ha llamado el poeta Chinweizu, la diferencia entre "The West and the Rest of Us" (el occidente y, nosotros, los demás" (Chinweizu). Sugiero que los patrones empleados hasta ahora, inclusive el de Bakhtin, no bastan para describir este fenómeno de la dominación de unas pocas naciones sobre muchas otras. Mientras su análisis ayuda mucho a comprender la división universal de clases dentro de una sociedad, el autor no anticipaba dicha división transnacional. Sugiero, más allá del análisis general que hace Harlow, que 1) entre los pueblos dominados están "las colonias internas" de los hispanos, los indígenas y los negros de los Estados Unidos diferencia que comprende la presencia de puertorriqueños también; 2) que existe una visión del mundo coloreada por el humor que se halla en la literatura de todos estos pueblos y 3) que a pesar de las distinciones, los de las clases alta y media en estos pueblos también pertenecen al tercer mundo

${ }^{2}$ El estudio de Harlow está en la vanguardia por su análisis de una base común para la temática y estructura de la literatura de los no poderosos. Sin embargo, la incorporación de los que muchos consideran colonias internas (i.e. los negros e hispanos de los Estados 
It may appear that when the outside world seems about to crush him completely, the writer has at least one possibility of escape: the creation of a humorous distance between himself and the world he is exposed to. But the humor of the Third World is not protection, nor escape. Like the humor of the disenchanted everywhere, it is purposeful. Ironic, bitter, even menacing, the humor of Third World writers is itself a weapon. It is used to identify the outrageous assumptions of racism and economic exploitation, the hypocrisy of governments, and to strengthen, cheer and even warn compatriots [...] The reader may smile or laugh aloud at the humor, but underneath the amusement, the razor-sharp message is inescapable (Troupe, xxxviii).

Volviendo a la idea de Harlow, de que dicha producción literaria representa un desafío a los códigos de la teoría y la crítica literaria, propongo la poética del humor como una característica en común entre los pueblos no poderosos. Esta asociación no busca negar las diferencias entre las culturas ni quiere hacer que no existan rasgos culturales-estéticos que identifiquen a cada pueblo y a cada autor como único. No niego la diferencia entre la escritora puertorriqueña y, por ejemplo, el trinitario Naipaul o el nigeriano Kole Omotoso. Decir "Vega" no es repetir el nombre de la afro-estadounidense Toni Morrison ni el del condenado indio Salman Rushdie. Además no quiero forzar la idea del humor, ya que no todos los autores de pueblos oprimidos tienen la misma capacidad ni interés en hacernos reír. De igual modo, hay muchos escritores de pueblos opresores cuyas obras son una verdadera delicia humorística. Sin embargo el humor define a gran parte de los autores tercermundistas cuyas obras tienen ese elemento de la mirada desde abajo que no tienen los escritores de pueblos poderosos. Lo que es más, no obstante las diferencias en la obra de los varios escritores tercermundistas que practican el humor, leemos la narración de culturas (y subculturas) en conflicto con las ideas dominantes sobre lo que es la cultura, la civilización y hasta lo que es el mundo occidental. Y muchas veces su narración es simplemente chistosa.

Se manifiestan dos tendencias generales en su desarrollo del humor. Cuando el énfasis está en la relación entre los oprimidos y los poderosos, el de abajo se presenta como gran changador, capaz de sobrevivir a pesar de las dificultades. En los momentos, como en la ficción de Vega, que hay un enfoque interno de los oprimidos, el personaje es el "changado" o sea el receptor de unas crueldades tremendas por parte del narrador.

Ethnic humor is presumed to have the function of intragroup social control when it directly or indirectly expresses approval of a group's identity and positive self-image for group members, and disapproves by ridicule individuals who do not conform to existing cultural norms or who wish to hide or deny their

Unidos, pero también, por ejemplo, los palestinos en el left bank) junto con el aislamiento de rasgos específicos de retórica como el humor, habrían agregado más a este estudio tan importante de la crítica. 
ethnic identity for personal gains. [...]In an ethnically pluralistic society, ethnic humor is more likely to be present among minority or subordinate groups, because of their need for social cohesion, than among majority or dominant groups who do not face the dilemma of choosing between acculturation and the maintenance of distinct ethnic identity (Apte, 143).

Para ser específica en la identificación de la fuente del humor en los cuentos de Vega es necesario conocer la región del Caribe. Una manifestación de la visión desde abajo descrita anteriormente, este enfoque humoresco, aparece en toda su obra, especialmente en Encancaranublado, cuya dedicatoria establece claramente el sueño de una hermandad entre los pueblos caribeños: "A la confederación caribeña del futuro para que llueva pronto y escampe". Este nacionalismo regional se expresa en ciertos reconocimientos y acontecimientos en la colección de cuentos. Dentro del contexto caribeño de su obra, he aquí algunos de los patrones que identifican a cada pueblo: los puertorriqueños revelan una lengua y una cultura -medio hispanoamericanos, medio estadounidenses. Los cubanos inmigrantes a Puerto Rico sufren perennemente de un complejo de superioridad entre los isleños. Los dominicanos padecen de la pobreza y la discriminación de otros isleños, y los haitianos sienten doblemente la opresión de los dominicanos.

Los cuentos que me interesan analizar son "Puerto Rican Syndrome", "Pollito Chicken", y "Letra para salsa". Tres narrativas que enfocan al puertorriqueño. Parece que la autora conserva su risa más audaz e hiperbólica para sus compatriotas. Sean isleños o neoyorquinos, los protagonistas en estos cuentos proyectan una visión bastante chistosa y negativa de la sociedad boricua.

De esa manera es posible mofarse de emigrantes que van de la isla a Nueva York e intentan ocultar y olvidar su identidad cultural. Así se burla de una sociedad entera que cree tanto en el sueño norteamericano que olvida quién es. Vega es igualmente capaz de burlarse de los muy machos que se creen totalmente superiores a la mujer.

¿Burla? ¿Realmente se trata de eso? Detrás de la risa que narcotiza al lector y le hace sentir que todo anda bien está el gran guayabo después del high. Si es el tipo de cuento que lo hace a uno reír a carcajadas, es como la risa del que se cae al piso del sillón de donde lee uno, gozando el chiste. Pero cuando piensa bien en lo que escribió la autora, se golpea duro con esa caída al piso. La puertorriqueña que se esconde detrás de las gafas, que rehúsa hablar su idioma, y que aspira a casarse con un WASP por su mera diferencia cultural es una realidad diaria para los isleños. Pero, a decir verdad, se trata de un deseo tristemente global entre los pueblos sin poder (Fanon, Captain-Hidalgo).

Cuando Susie Bermiúdez se casara porque maybe se casaría para pagar menos income tax - sería con un straight All-American, Republican, church-going, Wall-Street businessman, como su jefe Mister Bumper porque ésos sí que son good husbands y tratan a sus mujeres como real ladies criadas con el manual de Amy Vanderbilt y todo (Virgenes 76-77). 
Arrulla la risa por la facilidad con que la narradora es capaz de fluir entre el español y el inglés para describir los pensamientos de Susie. Su retrato realmente es chistoso y el lector comparte una guiñada con la narradora que convierte a este personaje en el hazmerreír de todos. Pensar casarse con un hombre que tenga todas las características contrarias a las que posee ella misma comprende un retrato tan insólito que ¿quién puede dejar de reírse? Pero, al pensarlo bien, se comprende que se identifica tanto con el pueblo ajeno que ha cambiado hasta su propio nombre. Ya no es quien era al nacer. Pero, a causa de su apellido, que en realidad simboliza su historia cultural, jamás llegará a ser quien aspira a ser.

En el sentido más amplio, lo que logra Ana Lydia Vega es forzar a su lector a pensar en los valores y aspiraciones de todo un sector de la sociedad. Por lo menos consigue Suzie Bermiúdez apreciar cierto aspecto de su cultura al encontrar su liberación física y gritar su apoyo para un Puerto Rico libre. Marca el principio de su conocimiento y auto-valorización y lo que el lector anticipa es una continuación política.

Al pensar en una sociedad latinoamericana y tercermundista en general, una de las percepciones, ya cliché, que viene a la mente del lector es la del machismo y el estado social inferior de la mujer. Pero cuando se termina el cuento "Letra para salsa", ya éste se siente obligado a re-ajustar la percepción de la mujer débil y sumisa. Igual que lo que logra su compatriota Rosario Ferré con su seriedad, Vega insiste en una nueva mujer, capaz de todo lo que antes era considerado dominio del hombre. Superficialmente, "Letra para salsa" abarca el tema de la igualdad mujer-hombre en la cama. Para el hombre en el cuento es un día típico en que trata de "coger" a una mujer con sus "piropos" habituales (habituales para la receptora, pero que él cree muy listos). El tipo, como es su nombre en la narración, encuentra a una mujer (la tipa) dispuesta a aceptar su oferta. Su igualdad entonces empieza a hacerse obvia desde un principio en sus nombres, que los nivelan y en su igual bravura. La rapidez con que consiente la mujer es sorprendente. Aun antes de llegar al lugar de su rendez-vous, el lector empieza a cuestionar la verdadera desigualdad de los dos. A pesar de sus palabras, el tipo está un poco reticente, y al llegar al hotel se sabe que no solamente es incapaz de costear el placer del coito, sino que tampoco puede cumplir, al principio por lo menos, las promesas hechas en la calle. De modo que se trata de una desigualdad en que la tipa es, irónicamente, superior.

Pero, después de reírse, ¿quién no confesará un reconocimiento de un problema que va mucho más allá de la igualdad genital? Materialmente la mujer está mucho más segura que el hombre. El tipo pertenece a todo un grupo de tipos-ociosos, jóvenes y viejos - plantados en las esquinas de San Juan y listos para bombardear a las mujeres con sus ofertas de un paraíso momentario. Al leer cierta literatura, los periódicos, o cualquier tipo de estudio sociológico, se conoce que es innegable también la asociación de estos hombres con otros que muchas veces ni siquiera pertenecen a la cultura latinoamericana. Pero sí son 
del tercermundo. Lo que intenta Vega, a través del humor es derrumbar primero todo estereotipo y mala costumbre, para luego empezar de nuevo. En el cuento "Puerto Rican Syndrome", qué chistoso, ¿verdad?, los niños que dan testimonio de su visión de la Virgen. Una imagen que, a propósito, va muy de acuerdo con una descripción de la más típica gringa que se ve en la televisiónimagen que se exporta a todo el mundo.

Estábamos mirando los muñequitos ... ENTONCES ... nos estábamos comiendo una caja Family Size de Rice Crispies ... ENTONCES ... dijo Pacheco que para ir al cielo había que ser doctor, arquitecto o abogado ... ENTONCES se oscureció la pantalla ... ENTONCES aparecio una señora bien linda, rubia, de ojos azules, vestida de rojo, azul y blanco y con el velo llenito de estrellas ... ENTONCES ... $(41-42)$

Aún más gracioso es el desarrollo de la creencia, cuando todo el pueblo puertorriqueño empieza a hacer su peregrinaje al barrio donde por primera vez aparecióla virgen. Claro es que la hiperbólica introducción de los investigadores de la WASP University agrega un toque de más humor al cuento. Sin embargo, tiene que venir el golpe de conciencia y el reconocimiento al pensar que el cuento "Puerto Rican Syndrome" es nada más que una alegoría de lo que ha ocurrido desde la llegada de los norteamericanos a la isla. En los casi cien años de neocolonización, la influencia anglosajona ha llegado a tal extremo que, como diría Luis Rafael Sánchez, es un virus, apenas perceptible, pero de todos modos peligroso. Esta influencia se acopla con la resistencia activa que, desde el primer instante de invasión cultural y política, ha protestado en varias formas.

Ana Lydia Vega examina a través de su cuentística la condición de oprimidos. Uno de los instrumentos con que se arma es el humor.

\section{BIBLIOGRAFIAA DE OBRAS CONSULTADAS}

Apte, Mahadev, L. Humor and Laughter: An Anthropological Approach Ithaca: Cornell University Press, 1985.

Aristotle. Aristotle's Poetics. Trans., James Hutton New York: Norton, 1982. Astroff, Roberta. "Communication and Contemporary Colonialism: Broadcast Television in Puerto Rico". Studies in Latin American Popular Culture 6. 1987.

Bakhtin, Mikhail. Rabelais and His World. Trans., Krystyna Pomorska Cambridge: MIT Press. 1968.

Barradas, Efraín. "La necesaria innovación de Ana Lydia Vega: preámbulo para lectores vírgenes". Revista Iberoamericana 132-33. Jul-Dic., 1985.

Captain-Hidalgo, Yvonne. “The Black Universal Continuum”. The Ennobling Cultural Discourse of Manuel Zapata Olivella (forthcoming).

Fanon, Frantz. Black Skin, White Masks . New York: Grove, 1967.

Harlow, Barbara. Resistance Literature . New York: Methuen, 1987. 
Jansen, William H. "The Esoteric-Exoterric Factor in Folklore". Fabula 2. (1959) 205-211.

Larson, Charles. R. The Novel in the Third World. Washington, D.C. INSCAPE, 1976.

Méndez, José, L. "Sobre vírgenes y mártires". Sin Nombre 14 (Oct-dic 1983) 61-67.

Rodríguez Monegal, Emir. “Tradition of Laughter”, Review: Latin American Literature and Arts 35. July-December, 1985.

Troupe, Quincy and Rainer Schute, ed. Giant Talk: An Anthology of Third World Writing. New York: Vintage, 1975.

Vega, Ana Lydia. Encancaranublado y otros cuentos de naufragio.. Río Piedras: Editorial Antillana, 1987.

Vega, Ana Lydia and Carmen Lugo Filippi. Vírgenes y mártires. Río Piedras: Editorial Antillana, 1983.

Vélez, Diana. "Pollito Chicken: Split Subjectivity, National Identity and the Articulation of Female Sexuality in a Narrative by Ana Lydia Vega". Americas Review 14 (Summer, 1986), 68-76.

Wolf-Phillips, L. "Why "Third World": Origin, Definition and Usage". Third World Quarterly 4 (Oct, 1987) 1311-1327. 